FERMILAB-TM-2608-CD

\title{
Simulation needs and priorities of the Fermilab Intensity Frontier experiments
}

\author{
V. D. Elvira, K. L. Genser, R. Hatcher, G. Perdue, H.J. Wenzel, J. Yarba \\ Fermi National Accelerator Laboratory, Batavia, IL, 60510
}

\begin{abstract}
Over a two-year period, the Physics and Detector Simulations (PDS) group of the Fermilab Scientific Computing Division (SCD), collected information from Fermilab Intensity Frontier experiments on their simulation needs and concerns. The process and results of these activities are documented here.
\end{abstract}

\section{Introduction}

Fermilab features a broad "Intensity Frontier" (IF) program including a comprehensive suite of experiments studying neutrinos, charged lepton flavor violation, the gyromagnetic ratio of the muon to unprecedented precision, and even nuclear structure. Each of the different areas comprising the overall program faces unique simulation challenges and all of these areas are addressed directly by the Physics and Detector Simulation (PDS) Group in the Scientific Computing Division at Fermilab. The PDS group is comprised of physicists and computer scientists that leverage their expertise through partnerships with experimenters working at the laboratory to produce important results despite modest personnel resources.

In addition to input from in-house scientists, the PDS group actively engages the IF community when considering its work-plan. PDS conducted a series of meetings with representatives from all the Fermilab IF experiments to discuss their simulation needs, and to define a strategy to improve the accuracy of detector and beam simulation tools used by the experiments. The initial meeting was held in April of 2012 with follow up discussions via e-mail and in meetings in early 2013 and 2014. In June 2014 more regular meetings, focusing on the needs of neutrino experiments were instituted and they have continued on a semi monthly basis since under the banner of "The Simulations for Neutrinos Working Group."

\section{Introduction to the simulation software in use on the Intensity Frontier}

The Intensity Frontier divides into three broad categories of experiments: neutrino, muon and nuclear structure. These share a common need to simulate the effects of particles in their detectors, but take different approaches for generating what the initial state was and their emphasis on what processes are important also differ.

Properly simulating a neutrino experiment like NOvA [14], MicroBooNE [8], MINERvA [9], or DUNE [6] involves a three-part software stack, factorized to isolate different 
physics challenges and concerns, as represented in Figure 1. The first part of the stack concerns the beamline and the neutrino flux prediction. Estimating the neutrino flux is a notorious problem because as weakly interacting particles, neutrinos offer no independent mechanisms for measuring the flux. The effluence of mesons coming off the target, which eventually decay to give neutrinos, is too intense to be measured in situ. A central problem is in the difficulty of accurately simulating the hadronic physics of meson production, especially in a thick target. Effects of focusing of the created mesons are also an important consideration. Geant4 [1] is an important software toolkit for this work, capable of simulating both the interactions in the target and the beamline itself. Some experiments use FLUKA [4] to make similar predictions with independent simulations of the physics processes, which allow for some measure of systematics estimation.

The second part of the neutrino software stack is the event generator. Information about the incoming energy is not available event-by-event in a neutrino experiment and so we must rely heavily on event generators to provide the probability-weighted mapping between observed final states and possible initial states. While there is a wealth of information available on nuclear effects and form-factors from charged lepton scattering, there is a dearth of similar information for neutrinos, making interaction physics an area of intense research and an important source of systematic uncertainty in a neutrino experiment. GENIE [2] is the event generator of choice for current and next generation neutrino experiments.

The final part of the stack is the detector simulation. Again there are numerous challenges in terms of efficiently simulating large numbers of events inside complex detector geometries and in handling subtle physics effects in the traversal of radiation across matter. Geant4 is the universal solution at this stage.

The muon-campus experiments, e.g. Muon g-2 [12] and Mu2e [13], also face large simulation challenges. In particular, because they are searching for extremely rare effects, they must grapple with very low rate background sources. Often the physics of these processes are not well understood, introducing large systematic effects. Additionally, the size of the computational problem is a serious challenge, as large simulation runs are required to adequately sample all the appropriate areas of experimental phase space, even when techniques to minimize the required computations are used. Geant 4 is the primary simulation toolkit for all stages of these experiments. 


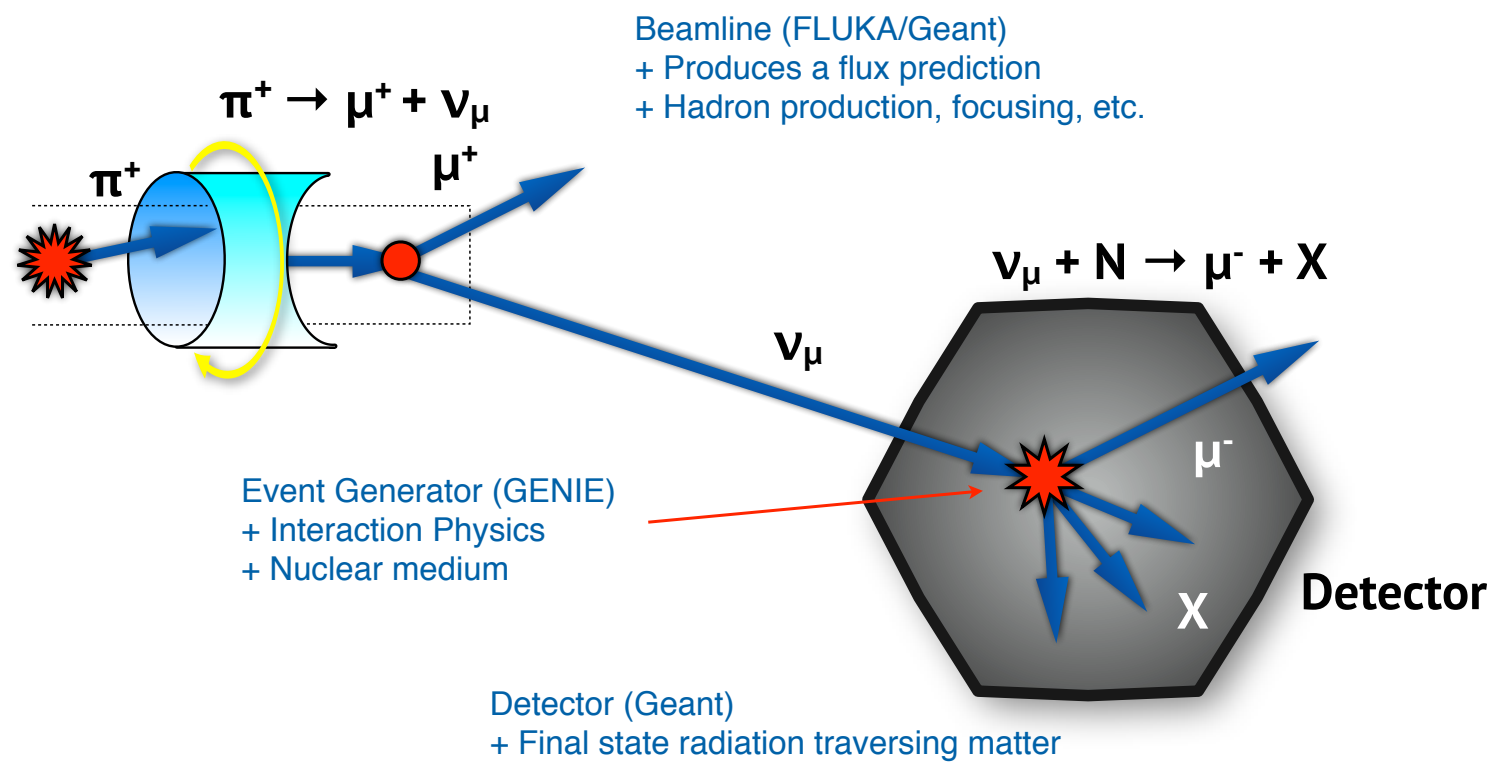

Figure 1: The three-part software stack of a neutrino experiment.

Test Beam experiments like LArIAT [7], which expose liquid Argon Time Projection Chambers (LArTPCs) to particle beams use predominantly Geant4 (within the LArSoft [16] framework).

Finally, fixed target nuclear structure experiments like SeaQuest [15] have their own unique challenges. Like a neutrino experiment, they must employ an event generator to address the tight coupling between the physics they are investigating and their simulation needs. In this case though, PYTHIA [3], also widely used in the Energy Frontier, is the generator of choice.

The PDS group is well suited to handle challenges across the full spectrum of these simulation activities; with membership in the Geant4, GENIE, and PYTHIA collaborations, the group has access to knowledge and domain-specific skills that are simply not available in most experimental collaborations. By working centrally at the laboratory the PDS group makes all of these resources available to the experimenters working there. By containing a mix of physicists engaged in active research at Fermilab and computer scientists pushing the boundaries of high performance computing, the PDS group is capable of understanding and effectively addressing the simulation needs at the IF.

\section{Community engagement in the design of a coherent work-plan: The process}

During a series of meetings between the PDS group and the experiments, representatives from DUNE (then LBNE), Muon g-2, MicroBooNE, MiniBooNE [10], MINOS/MINOS+ [11], MINERvA, Mu2e, NOvA, SeaQuest and (at a later stage) LArIAT all presented material on their greatest simulation challenges and needs. These representatives were appointed by the collaborations after invitations were sent to the spokespeople of the various groups. The representatives gave presentations outlining 
their concerns. Inclusive discussions including the PDS and the various experiments were conducted over the course of the meetings. All the interested parties were in the same room or connected by phone so there was ample opportunity for the experiments to share their concerns with the PDS and also learn from each other. There was time in between sessions for the representatives to return to their "home" experiments and iterate on findings and various issues. (The URLs of the meeting websites are available in the Appendix.)

\section{Community engagement in the design of a coherent work-plan: The results}

It was agreed that effort should be invested in validating existing Geant4 "physics lists" and eventually in developing customized lists for the intensity frontier experiments. Furthermore, the importance of performing validation and regression testing of new versions of the simulations vs. existing MC results was emphasized.

In addition to the general topics above, the following physics processes were specifically identified as important to the neutrino experiments:

- Hadronic showers following the first neutrino interaction in the range of several tens of $\mathrm{MeV}$ up to few $\mathrm{GeV}$.

- High-energy cosmic rays gradually going down to a few MeV.

- Muon polarization at the point of decay.

- Particle ID via dE/dx in liquid Argon.

- Proton stopping and $\mathrm{dE} / \mathrm{dx}$ profiles in liquid Argon.

- Hadronic and intra-nuclear scattering at low energy.

- Muon-nuclear interactions.

- Muon and antimuon separation in the absence of a magnetic field.

- Simulation of the interplay between ionization and scintillation light created when charge recombines.

- Pion and kaon identification utilizing their specific interactions and decay modes.

- Simulation of low energy electromagnetic showers and comparison with the experiment.

The following requests were also made:

- Enable adding "custom" features - for example, the ability to insert cross sections extracted from data at key points in the simulation.

- Enable model parameter tuning in order to understand systematic uncertainties on the model.

- Provide more detailed information on the physics lists and how to select the appropriate list for a given measurement. The liquid Argon neutrino experiments in particular sought guidance on how to assemble a list that could be shared across experiments, making studies in each experiment more useful and comparable to the others.

- Perform test of physics lists including studies of the model overlap regions. 
These high-level goals and requests were distilled into a series of action items and projects. The PDS group subsequently designed a series of internal work-plans and began publicly tracking progress on their Fermilab Redmine site:

https://cdcvs.fnal.gov/redmine/projects/stf

The details of these plans are presented in summary form below. Some of the items in the list are standing support issues and others are concrete projects with well-defined beginnings and ends.

\section{Geant4/GENIE usage related}

- Consultancy on best practices and advice on new features

- Consultancy related to migration to newer versions of Geant4

- Support of migration from various neutrino generator tools and Geant3 or FLUGG (FLUKA with Geant Geometry) to GENIE and Geant4

- Consultancy regarding Geant4 geometry

- Consultancy regarding Geant4 visualization tools

- Consultancy on Geant4 handling of step size, magnetic field parameters, handling of secondary particles

- Help with tau and charm decays in Geant4

- Development and support of a standardized art/Geant4 interface and a prototyping/validation tool (artG4Tk)

\section{Geant4/GENIE validation}

- Validation of Geant4 physics lists for beam and detector simulation with special emphasis on physics related to protons striking various target materials

- For neutrino and muon production and neutrino interactions and muon stopping/capture processes

- For processes generating electron backgrounds

The above involves collecting experimental data from experiments such as HARP, NA61, NA49, MIPP and performing simulations of protons striking thin or thick targets (e.g. C, Be, and perhaps Ta)

- Development and validation of new physics lists and Geant4 physics in partnership with experiments

- Development and support of GENIE validation tools

- Validation of Geant4 physics for liquid Argon - specifically to address the common concerns of the multiple neutrino experiments using Argon at Fermilab

- Extension of the validation datasets

- Validation of differences in muon and antimuon interactions (e.g. decay vs. capture) and the corresponding different detector response.

\section{Geant4/GENIE Development and Development Assistance}

- Create customized physics lists for simulation of beam-target interactions and detectors (including neutrino ones)

- Implement new processes related to Dark Matter production on target and interaction of Dark Matter with the detector in GENIE 
- Enable making (flux and detector response) predictions within an uncertainty band derived from known uncertainties in physics model parameters

- Cosmogenic simulation and validation, photon propagation lookup library

- Validate, and if needed improve or implement, processes generating backgrounds in the IF experiments (e.g. anti-proton production in case of Mu2e)

- Develop an API to allow GENIE to call hadronic physics libraries from within Geant4 for direct physics usage and to study systematic uncertainties.

- Develop an API to allow Geant4 to use GENIE to compute neutrino-nucleus cross sections.

- Develop tools to study ionization and scintillation light created in charge recombination. While this physics is included in LArSoft [16] it is not part of the standard Geant 4 distribution. This has implications for improving the energy resolution in LArTPCs.

\section{Geant4/GENIE Meeting Series}

It was resolved after the initial series of meetings to continue with a semi-regular (approximately monthly) meeting series aimed at the neutrino experiments at the laboratory. Because there are so many neutrino experiments and they share many common needs, the community felt a regular forum to share progress and highlight new concerns would be valuable.

\section{Conclusion}

Since the initial meetings took place, the PDS group has made significant progress on a number of high-level items. We have produced a new Geant 4 physics list for neutrino beamline simulations, designed a new GENIE validation application architecture and implemented the first example, and we have built a proof-of-principle application for studying model parameters to calculate systematic uncertainties in Geant 4 .

Perhaps most importantly we have established clear lines of communication with the experiments and are actively iterating on their needs and reacting to new information as the scientific program at Fermilab progresses and grows. By publicly tracking projects we are able to efficiently keep the entire community informed of the latest developments and we ensure continued relevancy and usefulness.

\section{References}

1. Geant4: A Simulation Toolkit, Nucl. Instrum. Meth. A506, 250-303, 2003

2. The GENIE Neutrino Monte Carlo Generator, Nucl. Intrum. Meth. A614, 87-104, 2010

3. PYTHIA 6.4 Physics and Manual, JHEP 0605, 026, 2006

4. FLUKA: a multi-particle transport code, CERN-2005-10 (2005), INFN/TC 05/11, SLAC-R-773 
5. ArgoNeuT, http://t962.fnal.gov

6. DUNE, http://www.dunescience.org

7. LArIAT, http://lariat.fnal.gov

8. MicroBooNE, http://www-microboone.fnal.gov

9. MINERvA, http://minerva.fnal.gov

10. MiniBooNE, http://www-boone.fnal.gov

11. MINOS/MINOS+, http://www-numi.fnal.gov/ http://www-numi.fnal.gov/MinosPlus/minosPlus.html

12. Muon g-2, http://muon-g-2.fnal.gov

13. Mu2e, http://mu2e.fnal.gov

14. NOvA, http://www-nova.fnal.gov

15. SeaQuest, http://www.phy.anl.gov/mep/seaquest/

16. LArSoft, https://cdcvs.fnal.gov/redmine/projects/larsoft

\section{Appendix: URLs of the PDS group meetings with the IF experiments}

The meeting agendas are available on the Fermilab Indico System, with presentations and minutes archived for future reference:

https://indico.fnal.gov/conferenceDisplay.py?confId=5499

https://indico.fnal.gov/conferenceDisplay.py?confId=6496

https://indico.fnal.gov/conferenceDisplay.py?confId=8147

https://indico.fnal.gov/conferenceDisplay.py?confId=8496

https://indico.fnal.gov/conferenceDisplay.py?confId=8581 